\title{
The natural history of rheumatic aortic regurgitation and the indications for surgery
}

Sir,

Smith et al. (1976) (British Heart fournal, 38, 147) in a recent article in the Journal painted a pessimistic picture of the prognosis in aortic regurgitation and consequently advocated early aortic valve replacement. This represented an opposite view to that expressed in two communications from our department (Goldschlager et al., 1973; Selzer, 1976). No one questions the poor prognosis of patients with serious disability, namely aortic regurgitation in functional classes III and IV. The point at issue is the management of patients without disability or with mild disability (functional classes I and II), who constituted about 50 per cent of those undergoing valve replacement in Smith et al.'s series (53 out of 110). In disabled patients valve replacement is a remedial operation; in those with minor or no disability it is largely a prophylactic operation.

Prophylactic operations require adequate knowledge of the natural history of the disease treated surgically in order to make a reasonable estimate of the risk-benefit ratio. This is particularly important in the case of valve replacement operations, in view of the uncertainty about the long range fate of the prosthesis or homograft and (in the case of aortic regurgitation) the young age of many patients. In the authors' series 22 patients died without operation; these patients showed a significantly higher incidence of heart failure, cardiomegaly (by radiography), excessive left ventricular hypertrophy by electrocardiography, and arrhythmias than survivors. This is not a finding that would surprise anyone; however, the number of fatal cases is small and no attempt has been made to analyse these 'unfavourable' signs as independent variables adversely affecting the prognosis. Thus, it is not valid to conclude that these findings represent indications for valve replacement.

It should be pointed out that the study by Goldschlager et al. (1973), which was a combined prospective and retrospective review of the natural history of aortic regurgitation based on clinical and haemodynamic evaluation, showed the benign and non-progressive nature of aortic regurgitation in young subjects. Cardiac failure and disability was seldom noted before the fifth decade of life. This finding not only was not refuted by the authors, but was not even mentioned, simply ignored. Yet Smith et al. report valve replacement in 70 patients under the age of 40 , out of 110 in their series.

It is also necessary to emphasize that the material examined by Smith et al. consisted of 31 per cent patients of Polynesian race. The presence of an accelerated form of rheumatic heart disease in the developing countries and primitive populations is now well established (Selzer and Cohn, 1972); the possible role of excessive early valve damage, its rapid progression, and continuation of active rheumatic carditis as causes for the unfavourable course of some patients has to be given serious consideration. It is likely that rheumatic aortic regurgitation may take a more benign course in Western populations.

The authors' advocacy for early valve replacement thus is based on opinion rather than reasonably projected or scientific evidence. Is it justified to place an aortic homograft valve in a 25 -year-old asymptomatic patient, because he happens to have a cardiothoracic ratio above 0.60 ? The authors apparently believe that it is; however, one would expect at least some reference to the fact that there is controversy on this point and that opposite views can be derived from other studies.
Arthur Selzer,
Division of Cardiology,
Presbyterian Hospital,
Pacific Medical Center,
San Francisco, CA 94120, USA.

\section{References}

Goldschlager, N., Pfeifer, J., Cohn, K., Popper, R., and Selzer, A. (1973). The natural history of aortic regurgitation. A clinical and hemodynamic study. American fournal of Medicine, 54, 577.

Selzer, A. (1976). Editorial. Cardiac valve replacement. An unanswered question. American fournal of Cardiology, 37, 322.

Selzer, A., and Cohn, K. (1972). Natural history of mitral stenosis. A review. Circulation, 45, 878. 
This letter was shown to the authors of the paper who reply as follows:

Sir,

It is not easy to reply to a critic who rejects the evidence on which the conclusions of a paper are based, simply by denying that it is evidence. But how does one decide if a factor, for example a cardiothoracic ratio of given magnitude, is associated with an unfavourable prognosis and should be an indication for aortic valve replacement? Presumably by showing a statistical association between the factor and death without operation, a higher incidence of complications at operation, or an unsatisfactory outcome despite a satisfactory operation. This was the aim of our paper and despite the relative crudity of the methods of assessment, correlation with outcome was surprisingly good. For example there was an increase in death rate when the cardiothoracic ratio reached $0 \cdot 60$. A cardiothoracic ratio of 0.65 or more was apparently associated with an increased incidence of unsatisfactory outcome after successful surgery, probably because of irreversible myocardial disease. We know of no other way to 'analyse these unfavourable signs as independent variables adversely affecting the 'prognosis'. We did, however, also attempt to assess the interrelation of the factors by extracting principal components and applying the technique of discriminant analysis, using an I.B.M. 360 computer programme. This analysis was carried out for us by Mr. C. D. McNickle of the Mathematics Department of the University of Auckland. These data were not reported because we concluded that a minimum of 2000 patients would be required for a valid assessment. Obviously the 'perfect' natural history study where large numbers of patients are followed to their death and analysed in this way will never be undertaken, and we are forced to base our judgments on the material available.

We must freely admit to having 'ignored' the report by Dr. Selzer and his colleagues, together with many other excellent studies on the subject of aortic regurgitation. Despite the wealth of detail in the study of Goldschlager et al. we did not feel that its design permitted conclusions about the timing of surgery for the following reasons:

(1) It included patients with moderate as well as those with severe aortic regurgitation. Because the prognosis of patients with moderate aortic regurgitation is extremely good we believe serious errors of interpretation arise from combining the two groups. Because of variation in systemic arteriolar resistance we do not believe the ratio of pulse pressure/systolic pressure, as used in Goldschlager's paper, separates moderate from severe regurgitation in a satisfactory fashion: indeed with the criteria employed, some patients in extremis with aortic regurgitation would have to be classified as mild. The difference in mortality of patients under observation in Goldschlager's group (6 per cent) and in our own group (12 per cent) probably results from the difference in selection. As mentioned in our paper, we found no evidence that Polynesians with isolated aortic regurgitation behaved differently from Caucasians though we could not deny the possibility that the factors operating 'in developing countries and primitive populations' might apply to our whole community!

(2) The paper did not consider the outcome of patients undergoing valve replacement.

We do not, of course, deny the prolonged survival of many patients with severe aortic regurgitation and indeed emphasised this in our paper. The gradual change in clinical status with time, emphasised by Goldschlager et al., is further shown in the Fig. In patients under observation without (or before) surgery the mean age of those classed as good was $21 \cdot 1$ years and of those classed as poor 37.8 years. It is noteworthy, however, that the mean age of those who died, 31.6 years, was similar to the overall mean of 30.6 years. In other words death occurred at any age and, furthermore, without regard to symptoms other than those labelled 'heart failure'.

In the interests of brevity the status of patients undergoing valve replacement was presented in somewhat perfunctory fashion. Although 10 patients were in Class I and 44 in Class II at the

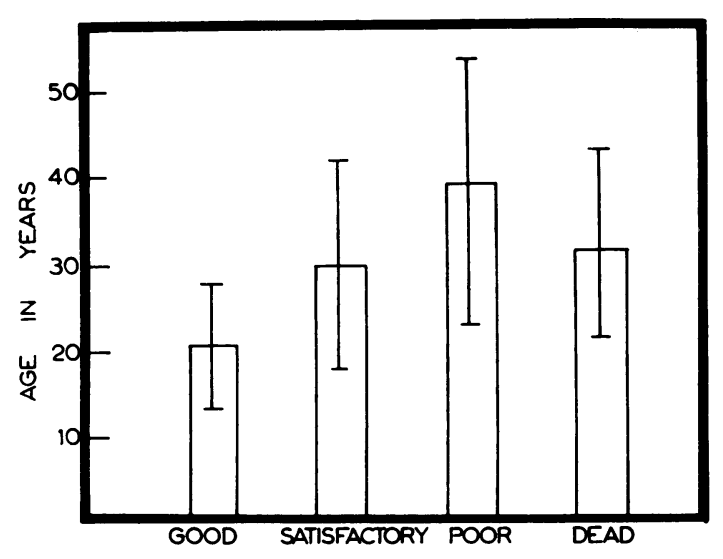

Fig. Relation between age and symptomatic status. Ages shown are means \pm 1 standard deviation. Good, class I; satisfactory, class II; poor, classes III and IV. 
time of preoperative assessment, many had experienced symptoms earlier but had responded to restriction of activities and to treatment with digitalis and diuretics. The limitations of this form of classification are well recognized (Selzer and Cohn, 1972). Even so there is a small group of patients who underwent aortic valve replacement in the mid 1960's in whom we would now postpone operation, even though the gain in most cases would only be a year or two. We emphasise that we agree entirely with Dr. Selzer that patients with aortic regurgitation should be managed conservatively as long as is compatible with reasonable safety. On the other hand, we must apply the lessons learned from those patients who have died while under observation, and attack the problems so well described by Goldschlager et al. 'The late ap- pearance of clinical disability at a stage when irreversible myocardial damage may be present imposes, at present, an insoluble therapeutic dilemma with regard to the timing of surgical treatment'.

H. J. Smith,

J. M. Neutze,

A. H. G. Roche,

T. M. Agnew,

B. G. Barratt-Boyes, Green Lane Hospital, Auckland, New Zealand.

\section{Reference}

Selzer, A., and Cohn, K. (1972). Functional classifications of cardiac disease: a critique. American fournal of Cardiology, 30, 306.

\section{World Congress of Cardiology}

The eighth World Congress of Cardiology will be held in Tokyo from 17 to 23 September 1978, at Hotel New Otani, Akasaka Prince Hotel, Tokyo, Japan.

Further information regarding registration, etc., can be obtained by writing to the Secretariat, Organizing Committee, VIII World Congress of Cardiology, 7-3-23, Roppongi, Minato-ku, Tokyo 106, Japan. 